TRANSACTIONS OF THE

AMERICAN MATHEMATICAL SOCIETY

Volume 356, Number 1, Pages 415-426

S 0002-9947(03)03347-6

Article electronically published on June 10, 2003

\title{
THE CLASSICAL PROBLEM OF THE CALCULUS OF VARIATIONS IN THE AUTONOMOUS CASE: RELAXATION AND LIPSCHITZIANITY OF SOLUTIONS
}

\author{
ARRIGO CELLINA
}

Abstract. We consider the problem of minimizing

$$
\int_{a}^{b} L\left(x(t), x^{\prime}(t)\right) d t, \quad x(a)=A, x(b)=B .
$$

Under the assumption that the Lagrangian $L$ is continuous and satisfies a growth assumption that does not imply superlinear growth, we provide a result on the relaxation of the functional and show that a solution to the minimum problem is Lipschitzian.

\section{INTRODUCTION}

The problem of minimizing

$$
\int_{0}^{1}(x(t))^{2}\left(x^{\prime}(t)\right)^{2} d t, \quad x(0)=0, x(1)=1,
$$

whose solution is $x(t)=\sqrt{t}$, provides a simple example of a variational problem having an integrand independent of the integration variable $t$, whose solutions are not Lipschitzian. The question of the regularity, in particular Lipschitzianity, of the solutions to a variational problem has long been of interest-Tonelli himself considered this problem. The purpose of the present paper is to prove the regularity (Lipschitzianity) of a solution $x$ to the problem of minimizing the functional

$$
\int_{a}^{b} L\left(x(t), x^{\prime}(t)\right) d t
$$

on the space of absolutely continuous functions $x():.[a, b] \rightarrow \mathbb{R}^{N}$ satisfying $x(a)=$ $A, x(b)=B$. The Lagrangian $L$ is independent of the integration variable $t$. Results implying the Lipschitzianity of the solution have been obtained in [B:M] and in $[\mathrm{C}: \mathrm{V}]$. These results are obtained under conditions of superlinear growth and of regularity for the Lagrangian. In this paper we obtain the Lipschitzianity of solutions under a very weak growth assumption, that does not imply superlinear growth, without assuming regularity of the Lagrangian (we assume continuity) and without assuming convexity of the Lagrangian with respect to its second variable.

Received by the editors September 4, 2001 and, in revised form, March 28, 2003.

2000 Mathematics Subject Classification. Primary 49N60.

Key words and phrases. Relaxation, regularity of solutions. 
As a side result, instrumental to the proof of the main theorem, we prove, under general assumptions, a relaxation result for functionals whose Lagrangian is (autonomous but) not convex in its second variable.

\section{NOTATION AND PRELIMINARY RESULTS}

By $B(\xi, r)$ and $B[\xi, r]$ we shall mean the open and, respectively, closed ball in $\mathbb{R}^{N}$, centered at $\xi$ and of radius $r . L^{*}(x, p)$ is the polar of $L$ with respect to its second variable, i.e., $L^{*}(x, p)=\sup _{\xi \in \mathbb{R}^{N}}\langle p, \xi\rangle-L(x, \xi)$, and $L^{* *}(x, \xi)$ is the bipolar of $L$ with respect to its second variable. In order that $L^{* *}$ be defined, a necessary and sufficient condition, to be assumed throughout, is that, for each $x, \xi \rightarrow L(x, \xi)$ be minorized by an affine function.

We shall call Problem $(P)$ the problem of minimizing

$$
\int_{a}^{b} L\left(x(t), x^{\prime}(t)\right) d t, \quad x(a)=A, x(b)=B,
$$

while Problem $\left(P^{* *}\right)$ is the problem of minimizing

$$
\int_{a}^{b} L^{* *}\left(x(t), x^{\prime}(t)\right) d t, \quad x(a)=A, x(b)=B .
$$

Definition. We shall say that the (not necessarily convex) function $f$ satisfies the Bounded Intersection Property $(B I P)$ if, for every $\tilde{\xi}$, there exists $\tilde{p} \in \partial f^{* *}(\tilde{\xi})$ such that

is bounded.

$$
\left\{\xi: f^{* *}(\xi)=f^{* *}(\tilde{\xi})+\langle\tilde{p}, \xi-\tilde{\xi}\rangle\right\}
$$

Theorem 1. Let $f$ be a continuous function, affinely minorized, satisfiying $(B I P)$. Then, for every $\tilde{\xi} \in \mathbb{R}^{N}$, there exist at most $\nu \leq N+1$ points $\xi_{i}$ and coefficients of a convex combination $\alpha_{i}$ such that

$$
\left(\begin{array}{c}
\tilde{\xi} \\
f^{* *}(\tilde{\xi})
\end{array}\right)=\sum_{0}^{\nu} \alpha_{i}\left(\begin{array}{c}
\xi_{i} \\
f\left(\xi_{i}\right)
\end{array}\right)
$$

Moreover, the $\xi_{i}$ belong to $\left\{\xi: f(\xi)=f^{* *}(\tilde{\xi})+\langle\tilde{p}, \xi-\tilde{\xi}\rangle\right\}$.

Proof. Fix $\tilde{\xi}$. Consider $\ell(\xi)=f^{* *}(\tilde{\xi})+\langle\tilde{p}, \xi-\tilde{\xi}\rangle$ such that the convex set $\{\xi$ : $\left.f^{* *}(\xi)=\ell(\xi)\right\}$ is bounded. Define $\psi: L \rightarrow \mathbb{R}$ by $\psi(\xi)=f(\xi)-\ell(\xi)$. The set $\{\xi \in L: \psi(\xi)=0\}$ is closed and contained in $\left\{\xi: f^{* *}(\xi)=\ell(\xi)\right\}$; hence it is compact. Then

$$
K=\operatorname{co}\{\xi: \psi(\xi)=0\}
$$

is compact. To prove the theorem, we are going to show that $\tilde{\xi} \in K$. Assume that $\tilde{\xi} \notin K$. Then $\tilde{\xi} \notin B[K, \delta]$ for some positive $\delta$, and so $\tilde{\xi}$ and the compact convex $B[K, \delta]$ can be strictly separated: there exist $a,\|a\|=1, \eta>0$ and $\xi^{0}$ such that $\left\langle a, \xi-\xi^{0}\right\rangle \leq 0$ on $B[K, \delta]$ and $\left\langle a, \tilde{\xi}-\xi^{0}\right\rangle=\eta$. Set $V_{n}=\left\{\xi: 0 \leq f^{* *}(\xi)-\ell(\xi) \leq\right.$ $\left.\frac{1}{n}\left\langle a, \xi-\xi^{0}\right\rangle\right\}$. Then $V_{n+1} \subset V_{n}$, and the $V_{n}$ are closed and convex. We claim that there exists $R$ such that, for $n$ large, we have $V_{n} \subset B[0, R]$. Otherwise, all the $V_{n}$ are unbounded and for each $n$ the set $D_{n}$ of the asymptotic directions $d_{n}:\left\|d_{n}\right\|=1$ $([\mathrm{R})$, is compact and nonempty. Let the nonempty set $D$ be their intersection. 
Then for any $d \in D$, any $n$ and $\lambda>0$,

$$
0 \leq f^{* *}(\lambda d)-\ell(\lambda d) \leq \frac{1}{n}\left\langle a, \lambda d-\xi^{0}\right\rangle,
$$

so that $f^{* *}(\lambda d)=\ell(\lambda d)$, contradicting $(B I P)$.

Hence there are $\tilde{n}$ and $R$ such that $n \geq \tilde{n}$ and $\|\xi\| \geq R$ imply that

$$
f^{* *}(\xi)-\ell(\xi)>\frac{1}{n}\left\langle a, \xi-\xi^{0}\right\rangle .
$$

There exists a positive $\varepsilon$ such that on the compact set $B[0, R] \backslash B(K, \delta)$ we have $\psi(\xi) \geq \varepsilon$. Let $n \geq \tilde{n}$ be such that

$$
\sup _{\{\|\xi\| \leq R\}} \frac{1}{n}\left\langle a, \xi-\xi^{0}\right\rangle \leq \frac{\varepsilon}{2} .
$$

We claim that the map $\ell(\xi)+\frac{1}{n}\left\langle a, \xi-\xi^{0}\right\rangle$ minorizes $f(\xi)$. This is certainly true when $\left\langle a, \xi-\xi^{0}\right\rangle \leq 0$; hence, in particular, when $\xi \in B[K, \delta]$, since $f(\xi)-\ell(\xi) \geq 0$. On the set $\{B[0, R] \backslash B(K, \delta)\}$, we have $\psi(\xi) \geq \varepsilon$, while $\frac{1}{n}\left\langle a, \xi-\xi^{0}\right\rangle \leq \frac{\varepsilon}{2}$; hence again $\ell(\xi)+\frac{1}{n}\left\langle a, \xi-\xi^{0}\right\rangle \leq f(\xi)$. Finally, when $\xi \in \mathbb{R}^{N} \backslash B[0, R]$,

$$
f(\xi) \geq f^{* *}(\xi)>\ell(\xi)+\frac{1}{n}\left\langle a, \xi-\xi^{0}\right\rangle
$$

proving the claim; hence, for every $\xi, f^{* *}(\xi) \geq \ell(\xi)+\frac{1}{n}\left\langle a, \xi-\xi^{0}\right\rangle$. However, we have

$$
\frac{1}{n}\left\langle a, \tilde{\xi}-\xi^{0}\right\rangle=\frac{1}{n} \eta
$$

hence we obtain $f^{* *}(\tilde{\xi}) \geq \ell(\tilde{\xi})+\frac{1}{n}\left\langle a, \tilde{\xi}-\xi^{0}\right\rangle=f^{* *}(\tilde{\xi})+\frac{1}{n} \eta$, a contradiction. Then $\tilde{\xi} \in \operatorname{coK}$, so that there are at most $\nu \leq N+1$ points $x_{i} \in K$ and coefficients of a convex combination $\alpha_{i}$ so that $\tilde{\xi}=\sum_{0}^{\nu} \alpha_{i} \xi_{i}$; moreover, since

$$
0=f^{* *}(\tilde{\xi})-\ell(\tilde{\xi})=\sum \alpha_{i}\left[f\left(\xi_{i}\right)-\ell\left(\xi_{i}\right)\right]=\sum \alpha_{i} f\left(\xi_{i}\right)-\ell(\tilde{\xi}),
$$

the theorem is proved.

Proposition 1. Let $f: \mathbb{R}^{N} \rightarrow \mathbb{R}$ be convex. The map $t \rightarrow\{\langle\xi t, p\rangle-f(\xi t): p \in$ $\partial f(\xi t)\}$, with values into the closed convex subsets of $\mathbb{R}$, is monotonically increasing.

Proof. This is proved in $\mathrm{C}: \mathrm{F}: \mathrm{M}$. The proof is easy when $f$ is smooth. In the general case it is obtained by approximating $f$ with smooth convex maps.

Proposition 2. Let $f: \mathbb{R}^{N} \rightarrow \mathbb{R}$ be convex. Then the function $(-1,+\infty) \ni \alpha \rightarrow$ $f\left(\frac{\xi}{1+\alpha}\right)(1+\alpha)$ is convex. Moreover, given $\delta$, there is $p \in \partial_{\xi} f\left(\frac{\xi}{1+\delta}\right)$ such that

$$
f\left(\frac{\xi}{1+\delta}\right)(1+\delta)-f(\xi) \leq-\delta f^{*}(p)
$$

Proof. The same as for the proof of Proposition 1.

The following Uniformity Lemma will be the key to obtaining global estimates from a pointwise growth assumption.

Uniformity Lemma. Let $f_{\zeta}($.$) be convex and let (\zeta, \xi) \rightarrow f_{\zeta}(\xi)$ be continuous. Assume that, for each $\zeta$ in a compact set $Z, \lim _{\|\xi\| \rightarrow \infty} f_{\zeta}^{*}(p(\xi))=+\infty$ for any selection $p($.$) from \partial f_{\zeta}($.$) . Then \lim _{\|\xi\| \rightarrow \infty} f_{\zeta}^{*}(p(\xi))=+\infty$, for any selection $p($. from $\partial f_{\zeta}($.$) , uniformly with respect to \zeta \in Z$. 
Proof. Otherwise, there exist $M$ and a sequence $\left(\zeta_{n}, \xi_{n}, p_{n}\left(\xi_{n}\right)\right)$, with $\zeta_{n} \in Z$, $\left\|\xi_{n}\right\| \rightarrow \infty$, and $p_{n}\left(\xi_{n}\right) \in \partial f_{\zeta_{n}}\left(\xi_{n}\right)$, such that $\left\langle\xi_{n}, p_{n}\left(\xi_{n}\right)\right\rangle-f_{\zeta_{n}}\left(\xi_{n}\right) \leq M$. We can assume that $\zeta_{n} \rightarrow \zeta^{*} \in Z$. Consider $\zeta^{*}$. There exists $R^{*}$ such that $\|\xi\| \geq R^{*}$ implies that

$$
\langle\xi, p(\xi)\rangle-f_{\zeta^{*}}(\xi)>M+1
$$

no matter what $p($.$) is in \partial f_{\zeta^{*}}($.$) . In particular,$

$$
\left\langle\frac{R^{*}}{\left\|\xi_{n}\right\|} \xi_{n}, p\left(\frac{R^{*}}{\left\|\xi_{n}\right\|} \xi_{n}\right)\right\rangle-f_{\zeta^{*}}\left(\frac{R^{*}}{\left\|\xi_{n}\right\|} \xi_{n}\right)>M+1,
$$

no matter how $p$ is chosen in $\partial f_{\zeta^{*}}\left(\frac{R^{*}}{\left\|\xi_{n}\right\|} \xi_{n}\right)$. Then

$$
\left.\left\langle\frac{R^{*}}{\left\|\xi_{n}\right\|} \xi_{n}, p\left(\frac{R^{*}}{\left\|\xi_{n}\right\|}\right) \xi_{n}\right)\right\rangle-f_{\zeta_{n}}\left(\frac{R^{*}}{\left\|\xi_{n}\right\|} \xi_{n}\right) \geq M+1+f_{\zeta^{*}}\left(\frac{R^{*}}{\left\|\xi_{n}\right\|} \xi_{n}\right)-f_{\zeta_{n}}\left(\frac{R^{*}}{\left\|\xi_{n}\right\|} \xi_{n}\right)
$$

and, from the uniform continuity of $f$ on $Z \times B\left[0, R^{*}\right]$, we find that there exists $\tilde{n}$ such that, for $n \geq \tilde{n}$,

$$
\left\langle\frac{R^{*}}{\left\|\xi_{n}\right\|} \xi_{n}, p\left(\frac{R^{*}}{\left\|\xi_{n}\right\|} \xi_{n}\right)\right\rangle-f_{\zeta_{n}}\left(\frac{R^{*}}{\left\|\xi_{n}\right\|} \xi_{n}\right)>M
$$

For any fixed sufficiently large $n$, apply Proposition 1 to the map $f=f_{\zeta_{n}}$, setting $t=\frac{\left\|\xi_{n}\right\|}{R^{*}}$, to infer that

$$
\begin{gathered}
\left\langle\xi_{n}, p_{n}\left(\xi_{n}\right)\right\rangle-f_{\zeta_{n}}\left(\xi_{n}\right)=\left\langle t \frac{R^{*}}{\left\|\xi_{n}\right\|} \xi_{n}, p_{n}\left(t \frac{R^{*}}{\left\|\xi_{n}\right\|} \xi_{n}\right)\right\rangle-f_{\zeta_{n}}\left(t \frac{R^{*}}{\left\|\xi_{n}\right\|} \xi_{n}\right) \\
\geq\left\langle\frac{R^{*}}{\left\|\xi_{n}\right\|} \xi_{n}, p\left(\frac{R^{*}}{\left\|\xi_{n}\right\|} \xi_{n}\right)\right\rangle-f_{\zeta_{n}}\left(\frac{R^{*}}{\left\|\xi_{n}\right\|} \xi_{n}\right)>M,
\end{gathered}
$$

a contradiction. Hence the lemma is proved.

Proposition 3. Let $F: \mathbb{R}^{N} \times \mathbb{R}^{N} \rightarrow \mathbb{R}$ be continuous and such that $\xi \rightarrow F(x, \xi)$ is convex, and consider the map $\Delta:(x, \xi) \rightarrow \partial_{\xi} F(x, \xi)$, with values in the compact subsets of $\mathbb{R}^{N}$.

i) $\Delta$ maps bounded sets into bounded sets.

ii) $\Delta$ is upper semicontinuous, i.e., for every $(x, \xi)$, for every $\varepsilon>0$ there exists $\delta>0$ such that

$$
\left\|x^{\prime}-x\right\|<\delta \text { and }\left\|\xi^{\prime}-\xi\right\|<\delta \text { imply } \partial_{\xi} F\left(x^{\prime}, \xi^{\prime}\right) \subset B\left[\partial_{\xi} F(x, \xi), \varepsilon\right] .
$$

In particular, the graph of $\Delta$ is closed.

Proof. i). If the claim is false, there exist $(x, \xi)$ and sequences $\left(x_{n}, \xi_{n}\right)$ and $\left(p_{n}\right)$, $p_{n} \in \partial_{\xi} F\left(x_{n}, \xi_{n}\right)$, such that $\left(x_{n}, \xi_{n}\right) \rightarrow(x, \xi)$ and $\left\|p_{n}\right\| \rightarrow+\infty$. Passing to a subsequence, we can assume that $\frac{p_{n}}{\left\|p_{n}\right\|} \rightarrow p_{0}$, where $\left\|p_{0}\right\|=1$, so that

$$
F\left(x, \xi+p_{0}\right)=\lim F\left(x_{n}, \xi_{n}+p_{0}\right) \geq \lim \sup \left[F\left(x_{n}, \xi_{n}\right)+\left\langle p_{n}, p_{0}\right\rangle\right]=+\infty,
$$

a contradiction to the continuity of $F$.

ii). If the claim is false, there exist $(x, \xi)$ and $\varepsilon>0$, and sequences $\left(x_{n}, \xi_{n}\right)$ and $\left(p_{n}\right), p_{n} \in \partial_{\xi} F\left(x_{n}, \xi_{n}\right)$, such that $\left(x_{n}, \xi_{n}\right) \rightarrow(x, \xi)$ and $d\left(p_{n}, \partial_{\xi} F(x, \xi)\right) \geq \varepsilon$. By i $)$, we can select a subsequence $p_{n} \rightarrow p_{*}$, and it follows that $p_{*} \notin \partial_{\xi} F(x, \xi)$. By the 
definition of subdifferential, there exists $\xi^{\prime}$ such that $F\left(x, \xi^{\prime}\right)<L(x, \xi)+\left\langle p_{*}, \xi^{\prime}-\xi\right\rangle$. Since

$$
\begin{aligned}
F\left(x, \xi^{\prime}\right)=\lim F\left(x_{n}, \xi^{\prime}\right) & \geq \limsup F\left(x_{n}, \xi_{n}\right)+\left\langle p_{n}, \xi^{\prime}-\xi_{n}\right\rangle \\
& =F\left(x, \xi^{\prime}\right)+\left\langle p_{*}, \xi^{\prime}-\xi\right\rangle,
\end{aligned}
$$

we have obtained a contradiction.

\section{THE GROWTH ASSUMPTION}

To obtain the main results of this paper, neither convexity nor differentiability of the Lagrangian with respect to $\xi$ will be assumed. Our results will depend on the validity of the growth assumption below: this assumption is expressed in terms of the convex (w.r.t. $\xi$ ) functions $L^{*}$ and $L^{* *}$. The function $\xi \rightarrow L^{* *}(x, \xi)$, being convex, admits a subdifferential, and we express the growth assumption in terms of this subdifferential.

For the special case of a bipolar $L^{* *}$ differentiable in $\xi$, the growth assumption simply states that, at $x, \lim _{\|\xi\| \rightarrow \infty} L^{*}\left(x, \nabla_{\xi} L^{* *}(x, \xi)\right)=+\infty$. Apart from the possible lack of differentiability of $L$, it is in any case convenient to express the growth assumption in terms of $\nabla_{\xi} L^{* *}$ instead of $\nabla_{\xi} L$ : in general, for a differentiable but non-convex function $f(\xi)$, the assumption $\lim _{\|\xi\| \rightarrow \infty} f^{*}\left(\nabla f^{* *}(\xi)\right)=+\infty$ is weaker than the assumption $\lim _{\|\xi\| \rightarrow \infty} f^{*}(\nabla f(\xi))=+\infty$, as the example of the function $t \rightarrow(2+\sin t) t^{2}$ shows: this function satisfies the first assumption but not the second.

Growth Assumption $(G A)$. The point $x$ statisfies $(G A)$ if for every selection $\xi \rightarrow p(x, \xi)$ from $\partial_{\xi} L^{* *}(x, \xi)$ we have

$$
\lim _{\|\xi\| \rightarrow \infty} L^{*}(x, p(x, \xi))=+\infty .
$$

We notice that $(G A)$ is a pointwise condition with respect to $x$.

Example. The convex map (already considered in $[\mathrm{C}: \mathrm{T}: \mathrm{Z}]$ ) $f: \mathbb{R} \rightarrow \mathbb{R}$ given by $f(t)=|t|-\sqrt{|t|}$ has linear growth, but $\lim _{|t| \rightarrow \infty}\left(t g^{\prime}(t)-g(t)\right)=\lim _{|t| \rightarrow \infty} \frac{1}{2} \sqrt{|t|}$ $=\infty$. To the contrary, the convex map $b: \mathbb{R} \rightarrow \mathbb{R}$, appearing in the Brachystocrone Problem, given by $b(t)=\sqrt{1+t^{2}}$, is such that $\lim _{|t| \rightarrow \infty}\left(t b^{\prime}(t)-b(t)\right)=0$.

The next proposition shows that convex maps (such as $\xi \rightarrow L^{* *}(x, \xi)$ ) with superlinear growth satisfy $(G A)$.

Proposition 4. Let $f: \mathbb{R}^{N} \rightarrow \mathbb{R}$ be a convex function such that $\lim _{\|\xi\| \rightarrow \infty} \frac{f(\xi)}{\|\xi\|}=$ $+\infty$. Then, for every selection $p(\xi)$ from $\partial f(\xi)$, we have $\lim _{\|\xi\| \rightarrow \infty}\langle\xi, p(\xi)\rangle-f(\xi)=$ $\lim _{\| \xi] \| \rightarrow \infty} f^{*}(p(\xi))=+\infty$.

Proof. Let $B$ be the unit ball of $\mathbb{R}^{N}$ and let $k$ be such that $f(x) \leq k$ for $x \in B$. For $x \in B$ and every $\xi$ we have

$$
k \geq f(x) \geq f(\xi)-\langle p(\xi), \xi\rangle+\langle p(\xi), x\rangle .
$$

Assume, to the contrary, that there exist a sequence $\left(\xi_{n}\right),\left\|\xi_{n}\right\| \rightarrow+\infty$, and $h$ such that $\left(\left\langle\xi_{n}, p\left(\xi_{n}\right)\right\rangle-f\left(\xi_{n}\right)\right) \leq h$. Then, along this sequence, $\left\|p\left(\xi_{n}\right)\right\| \leq k+h$. Since $f\left(\xi_{n}\right) \leq f(0)+\left\langle p\left(\xi_{n}\right), \xi_{n}\right\rangle$, we have obtained that

$$
f\left(\xi_{n}\right) \leq f(0)+(h+k)\left\|\xi_{n}\right\|,
$$

contradicting the superlinear growth of $f$. 
Theorem 2. Let $L: \mathbb{R}^{N} \times \mathbb{R}^{N} \rightarrow \mathbb{R}$ be continuous. Moreover, for each $x$ let $\xi \rightarrow L(x, \xi)$ be affinely minorized, and let $(x, \xi) \rightarrow L^{* *}(x, \xi)$ be continuous. Assume that every $x$ in a compact $C$ satisfies $(G A)$. Then:

i) $\lim _{\|\xi\| \rightarrow \infty} L^{*}(x, p(\xi))=+\infty$ for every selection $p(\xi)$ from $\partial_{\xi} L^{* *}(x, \xi)$, uniformly with respect to $x \in C$.

ii) Given $R_{0}>0$, there exists $R=R\left(R_{0}\right)$ such that for every $x$ in $C$, for every $\xi$, with $\|\xi\| \leq R_{0}$, there exist at most $\nu \leq N+1$ points $\xi_{i}$, with $\left\|\xi_{i}\right\| \leq R$, and coefficients of a convex combination $\alpha_{i}$ such that

$$
\left(\begin{array}{c}
\xi \\
L^{* *}(x, \xi)
\end{array}\right)=\sum_{0}^{\nu} \alpha_{i}\left(\begin{array}{c}
\xi_{i} \\
L\left(x, \xi_{i}\right)
\end{array}\right)
$$

Proof. We shall prove i) and ii) together.

a) Fix $R_{0}$. For fixed $x \in C, \tilde{\xi}$ and $\tilde{p} \in \partial L^{* *}(x, \tilde{\xi})$, set

$$
\ell_{x, \tilde{\xi}, \tilde{p}}(\xi)=L^{* *}(x, \tilde{\xi})+\langle\tilde{p}, \xi-\tilde{\xi}\rangle
$$

and define the function $\psi_{x, \tilde{\xi}, \tilde{p}}$ by

$$
\psi_{x, \tilde{\xi}, \tilde{p}}(\xi)=L^{* *}(x, \xi)-\ell_{x, \tilde{p}}(\xi)=L^{* *}(x, \xi)-L^{* *}(x, \tilde{\xi})-\langle\tilde{p}, \xi-\tilde{\xi}\rangle .
$$

It is a convex function of $\xi$ and it depends continuously on $(x, \tilde{\xi}, \tilde{p})$ and $\xi$. Moreover, $\partial \psi_{x, \tilde{\xi}, \tilde{p}}(\xi)=\partial_{\xi} L^{* *}(x, \xi)-\tilde{p}$. Consider the parameter set $Z=\left\{x \in C ;\|\tilde{\xi}\| \leq R_{0} ; \tilde{p} \in\right.$ $\left.\partial L^{* *}(x, \tilde{\xi})\right\}$; we claim that it is a compact subset of $\mathbb{R}^{N} \times \mathbb{R}^{N} \times \mathbb{R}^{N}$. In fact, apply Proposition 3 to the map $L^{* *}(x, \xi)$. By i), $Z$ is bounded. By ii), it is closed.

Fix $M>0$ arbitrarily. Apply the Uniformity Lemma to $\psi_{x, \tilde{\xi}, \tilde{p}}$ to infer the existence of $R$ such that $\|\xi\| \geq R$ implies that for any $x \in C$, any $\tilde{\xi}$, with $\|\tilde{\xi}\| \leq R_{0}$, and any $\tilde{p} \in \partial L^{* *}(x, \tilde{\xi})$, for every selection $p(\xi)$ from $\partial L_{\xi}^{* *}(x, \xi)$, we have

$$
\langle\xi, p(\xi)-\tilde{p}\rangle-\psi_{x, \tilde{\xi}, \tilde{p}}(\xi)>1,
$$

or, equivalently, $\|\xi\|\left\langle\frac{\xi}{\|\xi\|}, p\left(\|\xi\| \frac{\xi}{\|\xi\|}-\tilde{p}\right)\right\rangle-\psi_{x, \tilde{\xi}, \tilde{p}}\left(\|\xi\| \frac{\xi}{\|\xi\|}\right)>M$. In particular, this proves i).

b) Fix $\xi$ arbitrarily with $\|\xi\| \geq R$ and consider the convex function of the scalar $t$, $f(t)=\psi_{x, \tilde{\xi}, \tilde{p}}\left(t \frac{\xi}{\|\xi\|}\right)$ : we have $t p-f(t)>M$ for $t \geq R$, no matter what $p$ is in $\partial f(t)$, and $f(R) \geq 0$. In particular, $p(R) \geq \frac{M}{R}$ at $R$ and, for $t \geq R, f(t) \geq \frac{M}{R}(t-R)$. Equivalently, for every $\xi$ with $\|\xi\| \geq R, \psi_{x, \tilde{\xi}, \tilde{p}}(\xi)=\psi_{x, \tilde{\xi}, \tilde{p}}\left(\|\xi\| \frac{\xi}{\|\xi\|}\right) \geq \frac{M}{R}(\|\xi\|-R)$. Hence $L(x, \xi) \geq L^{* *}(x, \xi) \geq L^{* *}(x, \tilde{\xi})+\langle\tilde{p}, \xi-\tilde{\xi}\rangle$ for $\|\xi\| \leq R$ and $L(x, \xi) \geq$ $L^{* *}(x, \xi) \geq \frac{M}{R}(\|\xi\|-R)+L^{* *}(x, \tilde{\xi})+\langle\tilde{p}, \xi-\tilde{\xi}\rangle$ for $\|\xi\| \geq R$. Hence there is a convex function $C_{x, \tilde{\xi}, \tilde{p}}(\xi)$,

$$
C_{x, \tilde{\xi}, \tilde{p}}(\xi)= \begin{cases}L^{* *}(x, \tilde{\xi})+\langle\tilde{p}, \xi-\tilde{\xi}\rangle & \text { for }\|\xi\| \leq R, \\ \frac{M}{R}(\|\xi\|-R)+L^{* *}(x, \tilde{\xi})+\langle\tilde{p}, \xi-\tilde{\xi}\rangle & \text { otherwise }\end{cases}
$$

that minorizes $L$ and is such that $C_{x, \tilde{\xi}, \tilde{p}}(\xi)-\left[L^{* *}(x, \tilde{\xi})+\langle\tilde{p}, \xi-\tilde{\xi}\rangle\right]>0$ for $\|\xi\|>R$. By definition of $L^{* *}, L^{* *}(x, \xi) \geq C_{x, \tilde{\xi}, \tilde{p}}(\xi)$. Hence the set

$$
\left\{\xi: L^{* *}(x, \xi)=L^{* *}(x, \tilde{\xi})+\langle\tilde{p}, \xi-\tilde{\xi}\rangle\right\}
$$


is contained in $B[0, R]$ and the conclusion of Theorem 1 applies: for every $x \in C$, for every $\xi \in B\left[0, R_{0}\right]$, there are $\xi_{i},\left\|\xi_{i}\right\| \leq R$ and coefficients $\alpha_{i}$ such that

$$
\left(\begin{array}{c}
\xi \\
L^{* *}(x, \xi)
\end{array}\right)=\sum_{0}^{\nu} \alpha_{i}\left(\begin{array}{c}
\xi_{i} \\
L\left(x, \xi_{i}\right)
\end{array}\right)
$$

\section{RELAXATION}

Theorem 3. Let $L: \mathbb{R}^{N} \times \mathbb{R}^{N} \rightarrow \mathbb{R}$ be continuous. Moreover, for each $x$ let $\xi \rightarrow L(x, \xi)$ be affinely minorized, and let $(x, \xi) \rightarrow L^{* *}(x, \xi)$ be continuous.

i) Let $x():.[a, b] \rightarrow \mathbb{R}^{N}, x(a)=A, x(b)=B$, be absolutely continuous and assume that every $x(t), t \in[a, b]$, satisfies $(G A)$. Then for every $\varepsilon$ there exists $\tilde{x}($.$) ,$ depending on $\varepsilon$ and satisfying the boundary conditions, such that

$$
\int_{a}^{b} L\left(\tilde{x}(t), \tilde{x}^{\prime}(t)\right) d t \leq \int_{a}^{b} L^{* *}\left(x(t), x^{\prime}(t)\right) d t+\varepsilon .
$$

ii) Assume that every $x \in \mathbb{R}^{N}$ satisfies $(G A)$. Then

$$
\inf (P)=\inf \left(P^{* *}\right)
$$

Proof. i). Theorem 1 of [C:F:M], applied to the Lagrangian $L^{* *}$, assures that there exists a Lipschitzian function $x_{R_{0}}$, of Lipschitz constant $R_{0}$, satisfying the boundary conditions, such that

$$
\int_{a}^{b} L^{* *}\left(x_{R_{0}}(t), x_{R_{0}}^{\prime}(t)\right) d t \leq \int_{a}^{b} L^{* *}\left(x(t), x^{\prime}(t)\right) d t+\varepsilon / 2 .
$$

Moreover, $x_{R_{0}}($.$) is obtained from x($.$) through a reparametrization of the interval$ $[a, b]$, i.e., $\{x(t): t \in[a, b]\}=\left\{x_{R_{0}}(t): t \in[a, b]\right\}$ and every $x_{R_{0}}(t)$ with $t \in$ $[a, b]$ satisfies $(G A)$. Hence, to prove i), it is enough to show that there exists an absolutely continuous function $\tilde{x}($.$) , satisfying the boundary conditions, such that$

$$
\int_{a}^{b} L\left(\tilde{x}(t), \tilde{x}^{\prime}(t)\right) d t \leq \int_{a}^{b} L^{* *}\left(x_{R_{0}}(t), x_{R_{0}}^{\prime}(t)\right) d t+\varepsilon / 2
$$

Apply ii) of Theorem 2, where $C=\left\{x_{R_{0}}(t): t \in[a, b]\right\}$ : since $\left\|x_{R_{0}}^{\prime}(t)\right\| \leq R_{0}$ for a.e. $t$, we have that, for a.e. $t \in[a, b]$, there exist $R\left(R_{0}\right), \nu \leq N+1$, scalars $\alpha_{i}(t)$, and vectors $y_{i}(t)$, with $\left\|y_{i}(t)\right\| \leq R\left(R_{0}\right)$, such that

$$
\left(\begin{array}{c}
x_{R_{0}}^{\prime}(t) \\
L^{* *}\left(x_{R_{0}}(t), x_{R_{0}}^{\prime}(t)\right.
\end{array}\right)=\sum_{i=0, \ldots, \nu} \alpha_{i}\left(\begin{array}{c}
y_{i}(t) \\
L\left(x_{R_{0}}(t), y_{i}(t)\right)
\end{array}\right) .
$$

By a standard measurable selection argument, as in [E:T], we can assume that the maps $y_{i}($.$) and \alpha_{i}($.$) are measurable. Hence the maps y_{i}($.$) and L\left(x_{R_{0}}(),. y_{i}().\right)$ are integrable.

Fix an integer $m$ and consider the intervals $I_{j}=\left[a+j \frac{b-a}{m}, a+(j+1) \frac{b-a}{m}\right)$, $j=0, \ldots, m-1$, and denote by $\chi_{j}$ their characteristic functions. By Liapunov's theorem on the range of vector measures (as in [Ce]), there exists a partition of $[a, b]$ into $\nu$ measurable subsets $E_{i}$, with characteristic functions $\chi_{i}$, such that, for 
$j=1, \ldots, m$, one has

$$
\begin{aligned}
& \int_{[a, b]}\left(\begin{array}{c}
x_{R_{0}}^{\prime}(t) \\
L^{* *}\left(x_{R_{0}}(t), x_{R_{0}}^{\prime}(t)\right)
\end{array}\right) \chi_{j}(t) d t \\
& =\int_{[a, b]}\left[\sum_{i=0, \ldots, \nu} \alpha_{i}(t)\left(\begin{array}{c}
y_{i}(t) \\
L\left(x_{R_{0}}(t), y_{i}(t)\right)
\end{array}\right)\right] \chi_{j}(t) d t \\
& =\int_{[a, b]}\left[\sum_{i=0, \ldots, \nu} \chi_{i}(t)\left(\begin{array}{c}
y_{i}(t) \\
L\left(x_{R_{0}}(t), y_{i}(t)\right)
\end{array}\right)\right] \chi_{j}(t) d t .
\end{aligned}
$$

Denote by $x_{m}$ the absolutely continuous function defined by $x_{m}(a)=A$ and $x_{m}^{\prime}(t)=\int_{a}^{t} \sum_{j=0, \ldots, m-1} \sum_{i=0, \ldots, \nu} y_{i}(s) \chi_{j}(s) \chi_{i}(s) d s$; in particular, for every $m$ and every $j=1, \ldots, m$, we have

$$
\int_{I_{j}} x_{R_{0}}^{\prime}(t) d t=\int_{I_{j}} x_{m}^{\prime}(t) d t
$$

so that the functions $x_{R_{0}}$ and $x_{m}$ coincide at each point $a+j \frac{b-a}{m}$. Since

$$
\begin{aligned}
& \sum_{j=0, \ldots, m-1} \sum_{i=0, \ldots, \nu} \chi_{i}(t) \chi_{j}(t) L\left(x_{R_{0}}(t), y_{i}(t)\right) \\
= & L\left(x_{R_{0}}(t), \sum_{j=0, \ldots, m-1} \sum_{i=0, \ldots, \nu} \chi_{i}(t) \chi_{j}(t) y_{i}(t)\right),
\end{aligned}
$$

we also have

$$
\begin{aligned}
\int_{[a, b]} L^{* *}\left(x_{R_{0}}(t), x_{R_{0}}^{\prime}(t)\right) d t= & \int_{[a, b]} L\left(x_{R_{0}}(t), x_{m}^{\prime}(t)\right) d t \\
= & \int_{[a, b]} L\left(x_{m}(t), x_{m}^{\prime}(t)\right) d t \\
& +\int_{[a, b]}\left[L\left(x_{R_{0}}(t), x_{m}^{\prime}(t)\right)-L\left(x_{m}(t), x_{m}^{\prime}(t)\right)\right] d t .
\end{aligned}
$$

Since $x_{m}^{\prime}(t)$, for a.e. $t$, is contained in $B\left[0, R\left(R_{0}\right)\right]$ and $\left\{x_{R_{0}}(t): t \in[a, b]\right\}$ is compact, there exists $\sigma>0$ such that $\left\|\xi-x_{R_{0}}(t)\right\| \leq \sigma$ implies

$$
\left|L\left(x_{R_{0}}(t), x_{m}^{\prime}(t)\right)-L\left(\xi, x_{m}^{\prime}(t)\right)\right| \leq \frac{\varepsilon}{b-a} .
$$

Since $x_{R_{0}}$ is uniformly continuous on $[a, b]$ and the $x_{m}$ are equi-Lipschitzian and agree with $x_{R_{0}}$ at the points $a+j \frac{b-a}{m}$, by choosing $m$ large enough we obtain that $\left\|x_{m}(t)-x_{R_{0}}(t)\right\| \leq \sigma$, every $t \in[a, b]$; thus any $x_{m}$ with $m$ sufficiently large can serve as the desired function $x$.

ii). Since $L(x, \xi) \geq L^{* *}(x, \xi)$, it is enough to show that, given $x($.$) and \varepsilon>0$, there exists $\tilde{x}($.$) , satisfying the boundary conditions, such that$

$$
\int_{a}^{b} L\left(\tilde{x}(t), \tilde{x}^{\prime}(t)\right) d t \leq \int_{a}^{b} L^{* *}\left(x(t), x^{\prime}(t)\right) d t+\varepsilon .
$$

The existence of $\tilde{x}($.$) follows from i).$ 


\section{LIPSCHITZIANITY OF SOLUTIONS}

The purpose of this section is to prove the following theorem, the main result of this paper.

Theorem 4. Let $L: \mathbb{R}^{N} \times \mathbb{R}^{N} \rightarrow \mathbb{R}$ be continuous. Moreover, for each $x$ let $\xi \rightarrow L(x, \xi)$ be affinely minorized, and let $(x, \xi) \rightarrow L^{* *}(x, \xi)$ be continuous. Let $x($. be a solution to Problem $(P)$ such that $(G A)$ is satisfied at every $x(t), t \in[a, b]$. Then $x($.$) is Lipschitzian.$

To compare Theorem 4 with previously known results, notice that in [C:V] the Lipschitzianity of a solution is proved under the assumptions that i) $\xi \rightarrow L(x, \xi)$ is convex, ii) $L$ is locally Lipschitzian jointly in $(x, \xi)$, and iii) there exists a convex function $\theta:[0, \infty \rightarrow \mathbb{R})$ such that $\lim _{r \rightarrow+\infty} \frac{\theta(r)}{r}=+\infty$ and

$$
L(x, \xi) \geq \theta(\|\xi\|) .
$$

Proof of Theorem 4. a) Set $K=\int_{[a, b]}\left\|x^{\prime}(s)\right\| d s ; M=\frac{K}{b-a}$. There exists a measurable set $S^{M} \subset I$ of positive measure such that $s \in S^{M}$ implies $\left\|x^{\prime}(s)\right\| \leq M$.

Let $C=\{x(t): t \in[a, b]\}$, a compact subset of $\mathbb{R}^{N}$. The map $L^{* *}(x, \xi)$ is uniformly continuous on $C \times B[0,2 M]$. Fix $\eta>0$, there exists $\delta, 0<\delta \leq \frac{1}{2}$, such that

$$
\left|L^{* *}\left(x, \frac{\xi_{0}}{1-\delta}\right)(1-\delta)-L^{* *}\left(x, \xi_{0}\right)\right|<\eta
$$

for every $\left(x, \xi_{0}\right) \in C \times B[0, M]$.

Apply i) of Theorem 2 to infer that there exists $\Lambda$ such that $\frac{\left\|\xi_{1}\right\|}{1+\delta} \geq \Lambda$ implies that $L^{*}(x, p) \geq \frac{2 \eta}{\delta}$, for any $p \in \partial_{\xi} L^{* *}\left(x, \xi_{1}\right)$ and any $x \in C$.

Consider $S_{\Lambda} \stackrel{\delta}{=}\left\{t \in[a, b]:\left\|x^{\prime}(t)\right\| \geq \Lambda(1+\delta)\right\}$. To prove the theorem we shall show that the assumption that the set $S_{\Lambda}$ has positive measure leads to a contradiction. More precisely, assuming the measure of $S_{\Lambda}$ to be positive, in steps b) through f) we shall show the existence of a positive real $\sigma$ and of an absolutely continuous function, denoted $x_{\tilde{\varepsilon}}($.$) , obtained from x($.$) by a reparametrization of$ the interval $[a, b]$, such that

$$
\int_{a}^{b} L^{* *}\left(x_{\tilde{\varepsilon}}(t), x_{\tilde{\varepsilon}}^{\prime}(t)\right) d t \leq \int_{a}^{b} L\left(x(s), x^{\prime}(s)\right) d s-\sigma .
$$

In step g), applying Theorem 3, we shall show that this contradicts the fact that $x($.$) is a solution to (P)$.

b) From Proposition 2 , given $x$ and $\xi_{1}$, there exists $p \in \partial_{\xi} L^{* *}\left(x, \frac{\xi_{1}}{1+\delta}\right)$ such that

$$
L^{* *}\left(x, \frac{\xi_{1}}{1+\delta}\right)(1+\delta)-L^{* *}\left(x, \xi_{1}\right) \leq-\delta L^{*}(x, p)
$$

hence, whenever $x \in C$ and $\left\|\frac{\xi_{1}}{1+\delta}\right\| \geq \Lambda$, we infer that

$$
L^{* *}\left(x, \frac{\xi_{1}}{1+\delta}\right)(1+\delta)-L^{* *}\left(x, \xi_{1}\right) \leq-2 \eta .
$$

c) Consider $S^{M}$, defined in a), and let $\chi_{M}$ be its characteristic function: a.e. $t \in S^{M}$ is a point of density of $S^{M}$ and a Lebesgue point for the maps $t \rightarrow$ $L^{* *}\left(x(t), x^{\prime}(t)\right)$ and $t \rightarrow L^{* *}\left(x(t), \frac{x^{\prime}(t)}{1-\delta \chi_{M}(t)}\right)\left(1-\delta \chi_{M}(t)\right)$. Let $t^{0}$ be one such point. 
For a natural $\nu$, set $S^{\nu}=\left\{t:\left\|x^{\prime}(t)\right\| \leq \nu\right\}$; when $\nu$ is sufficiently large, if the measure of $S_{\Lambda}$ is positive, also the measure of $S_{\Lambda}^{\nu}=S_{\Lambda} \cap S^{\nu}$ is positive. Fix one such $\nu$ and let $\chi_{\Lambda}^{\nu}$ be the characteristic function of $S_{\Lambda}^{\nu}$. The map

$$
t \rightarrow L^{* *}\left(x(t), \frac{x^{\prime}(t)}{1+\delta \chi_{\Lambda}^{\nu}(t)}\right)\left(1+\delta \chi_{\Lambda}^{\nu}(t)\right)
$$

is integrable; a.e. $t \in S_{\Lambda}^{\nu}$ is a point of density and a Lebesgue point for the maps $t \rightarrow L^{* *}\left(x(t), x^{\prime}(t)\right)$ and $t \rightarrow L^{* *}\left(x(t), \frac{x^{\prime}(t)}{1+\delta \chi_{\Lambda}^{\nu}(t)}\right)\left(1+\delta \chi_{\Lambda}^{\nu}(t)\right)$. Let $t^{1}$ be one such point. We can assume that both $t^{0}$ and $t^{1}$ are in $(a, b)$.

For $\varepsilon$ sufficiently small, set $r_{0}(\varepsilon)=\int_{t^{0}}^{t^{0}+\varepsilon} \chi_{M}(t) d t$; we have $\lim _{\varepsilon \rightarrow 0} \frac{r_{0}(\varepsilon)}{\varepsilon}=1$. Set also

$$
\varepsilon_{1}(\varepsilon)=\inf \left\{\eta>0: \int_{t^{1}-\eta}^{t^{1}} \chi_{\Lambda}^{\nu}(t) d t=r^{0}(\varepsilon)\right\}
$$

so that, in particular, $\int_{t^{1}-\varepsilon_{1}(\varepsilon)}^{t^{1}} \chi_{\Lambda}^{\nu}(t) d t=r^{0}(\varepsilon)=\int_{t^{0}}^{t^{0}+\varepsilon} \chi_{M}(t) d t$. About the (monotonic nondecreasing) function $\varepsilon_{1}(\varepsilon)$ we have:

i) $\lim _{\varepsilon \rightarrow 0} \varepsilon_{1}(\varepsilon)=0$.

In fact, otherwise, there exists $\lambda>0$ such that $\varepsilon_{1}(\varepsilon) \geq \lambda$ for all $\varepsilon$, and from $\int_{t^{1}-\lambda}^{t^{1}} \chi_{\Lambda}^{\nu}(t) d t \leq r^{0}(\varepsilon)$ for all $\varepsilon$, we would infer that $\int_{t^{1}-\lambda}^{t^{1}} \chi_{\Lambda}^{\nu}(t) d t=0$, a contradiction, since $t$ is a point of density of $S_{\Lambda}^{\nu}$.

Moreover, we have

ii) $\lim _{\varepsilon \rightarrow 0} \frac{\varepsilon_{1}(\varepsilon)}{\varepsilon}=1$.

In fact, from i) above, since $t^{1}$ is a point of density of $S_{\Lambda}^{\nu}$,

$$
1=\lim _{\varepsilon \rightarrow 0} \frac{\varepsilon_{1}(\varepsilon)}{\int_{t^{1}-\varepsilon_{1}(\varepsilon)}^{t^{1}} \chi_{\Lambda}^{\nu}(t) d t}=\lim _{\varepsilon \rightarrow 0} \frac{\varepsilon_{1}(\varepsilon)}{r^{0}(\varepsilon)}=\lim _{\varepsilon \rightarrow 0} \frac{\varepsilon_{1}(\varepsilon)}{\varepsilon} \frac{\varepsilon}{r^{0}(\varepsilon)}
$$

since $\frac{\varepsilon}{r^{0}(\varepsilon)} \rightarrow 1$, this proves ii).

d) For all sufficiently small $\varepsilon$, consider the family of absolutely continuous maps $t_{\varepsilon}(s)$ defined by $t_{\varepsilon}(a)=a$ and

$$
t_{\varepsilon}^{\prime}(s)= \begin{cases}1+\delta \chi_{\Lambda}^{\nu}(t) & \text { if } t \in\left[t^{1}-\varepsilon_{1}(\varepsilon), t^{1}\right] \\ 1-\delta \chi_{M}(t) & \text { if } t \in\left[t^{0}, t^{0}+\varepsilon\right] \\ 1 & \text { otherwise. }\end{cases}
$$

In particular, it follows that, for every $\varepsilon>0$ sufficiently small, $t_{\varepsilon}$ is a Lipschitzian monotonic increasing transformation, mapping $[a, b]$ onto itself. From the properties of $t^{0}$ and $t^{1}$ we have

$$
\begin{gathered}
\int_{a}^{b} L^{* *}\left(x(s), \frac{x^{\prime}(s)}{t_{\varepsilon}^{\prime}(s)}\right)\left(t_{\varepsilon}^{\prime}(s)\right) d s-\int_{a}^{b} L^{* *}\left(x(s), x^{\prime}(s)\right) d s \\
=\int_{t^{0}}^{t^{0}+\varepsilon} L^{* *}\left(x(s), \frac{x^{\prime}(s)}{1-\delta \chi_{M}(s)}\right)\left(1-\delta \chi_{M}(s)\right) d s-\int_{t^{0}}^{t^{0}+\varepsilon} L^{* *}\left(x(s), x^{\prime}(s)\right) d s \\
+\int_{t^{1}-\varepsilon_{1}(\varepsilon)}^{t^{1}} L^{* *}\left(x(s), \frac{x^{\prime}(s)}{1+\delta \chi_{\Lambda}^{\nu}(s)}\right)\left(1+\delta \chi_{\Lambda}^{\nu}(s)\right) d s-\int_{t^{1}-\varepsilon_{1}(\varepsilon)}^{t^{1}} L^{* *}\left(x(s), x^{\prime}(s)\right) d s
\end{gathered}
$$




$$
\begin{gathered}
=\varepsilon\left\{L^{* *}\left(x\left(t^{0}\right), \frac{x^{\prime}\left(t^{0}\right)}{1-\delta}\right)(1-\delta)-L^{* *}\left(x\left(t^{0}\right), x^{\prime}\left(t^{0}\right)\right)+h^{0}(\varepsilon)\right\} \\
+\varepsilon_{1}(\varepsilon)\left\{L^{* *}\left(x\left(t^{1}\right), \frac{x^{\prime}\left(t^{1}\right)}{1+\delta}\right)(1+\delta)-L\left(x\left(t^{1}\right), x^{\prime}\left(t^{1}\right)\right)+h^{1}\left(\varepsilon_{1}(\varepsilon)\right)\right\},
\end{gathered}
$$

where $\lim _{\varepsilon \rightarrow 0} h^{0}(\varepsilon)=\lim _{\varepsilon \rightarrow 0} h^{1}(\varepsilon)=0$.

e) Since $\left\|x^{\prime}\left(t^{0}\right)\right\| \leq M$ and $\left\|x^{\prime}\left(t^{1}\right)\right\| \geq \Lambda(1+\delta)$, from the estimates obtained in a) and b) we obtain

$$
\begin{aligned}
\int_{a}^{b} L^{* *}\left(x(s), \frac{\left.x^{\prime}(s)\right)}{t_{\varepsilon}^{\prime}(s)}\right)\left(t_{\varepsilon}^{\prime}(s)\right) d s-\int_{a}^{b} L^{* *}\left(x(s), x^{\prime}(s)\right) d s \\
\quad \leq \varepsilon\left[\eta+h^{0}(\varepsilon)\right]+\varepsilon_{1}(\varepsilon)\left[-2 \eta+h^{1}\left(\varepsilon_{1}(\varepsilon)\right)\right] \\
\quad=\varepsilon\left[\eta-2 \eta \frac{\varepsilon_{1}(\varepsilon)}{\varepsilon}+h^{0}(\varepsilon)+\frac{\varepsilon_{1}(\varepsilon)}{\varepsilon} h^{1}\left(\varepsilon_{1}(\varepsilon)\right)\right] .
\end{aligned}
$$

f) Consider the family of maps $s_{\varepsilon}(t)$, where $s_{\varepsilon}(t)$ is the inverse of the map $t_{\varepsilon}(s)$, and set $x_{\varepsilon}(t)=x\left(s_{\varepsilon}(t)\right)$; since $s_{\varepsilon}$ is Lipschitzian and $x$ is absolutely continuous, $x_{\varepsilon}$ is an absolutely continuous map satisfying the boundary conditions. The chain rule for derivatives holds $[\mathrm{S}: \mathrm{V}]$, and we have

$$
\begin{aligned}
\int_{a}^{b} L^{* *}\left(x_{\varepsilon}(t), x_{\varepsilon}^{\prime}(t)\right) d t & =\int_{a}^{b} L^{* *}\left(x_{\varepsilon}\left(t_{\varepsilon}(s)\right),\left(\frac{d}{d t} x_{\varepsilon}\right)\left(t_{\varepsilon}(s)\right)\right) t_{\varepsilon}^{\prime}(s) d s \\
& =\int_{a}^{b} L^{* *}\left(x(s), \frac{x^{\prime}(s)}{t_{\varepsilon}^{\prime}(s)}\right) t_{\varepsilon}^{\prime}(s) d s .
\end{aligned}
$$

Recalling that $L(x, \xi) \geq L^{* *}(x, \xi)$, we see that the conclusion of e) gives

$$
\begin{aligned}
& \int_{a}^{b} L^{* *}\left(x_{\varepsilon}(t), x_{\varepsilon}^{\prime}(t)\right) d t-\int_{a}^{b} L\left(x(s), x^{\prime}(s)\right) d s \\
& \leq \varepsilon\left[\eta-2 \eta \frac{\varepsilon_{1}(\varepsilon)}{\varepsilon}+h^{0}(\varepsilon)+\frac{\varepsilon_{1}(\varepsilon)}{\varepsilon} h^{1}\left(\varepsilon_{1}(\varepsilon)\right)\right],
\end{aligned}
$$

i.e., there exists $\tilde{\varepsilon}$ such that

$$
\int_{a}^{b} L^{* *}\left(x_{\tilde{\varepsilon}}(t), x_{\tilde{\varepsilon}}^{\prime}(t)\right) d t-\int_{a}^{b} L\left(x(s), x^{\prime}(s)\right) d s \leq-\tilde{\varepsilon} \eta / 2=-\sigma .
$$

g) In the statement of Theorem 3 take $x($.$) to be x_{\tilde{\varepsilon}}(),. \varepsilon$ to be $\sigma / 2$. The set $C$ defined in a) coincides with $\left\{x_{\tilde{\varepsilon}}(t): t \in[a, b]\right\}$, so that $(G A)$ holds on $\left\{x_{\tilde{\varepsilon}}(t): t \in[a, b]\right\}$. Applying i) of Theorem 3, we infer the existence of $\tilde{x}($.$) ,$ satisfying the boundary conditions, such that

$$
\int_{a}^{b} L\left(\tilde{x}(t), \tilde{x}^{\prime}(t)\right) d t \leq \int_{a}^{b} L^{* *}\left(x_{\tilde{\varepsilon}}(t), x_{\tilde{\varepsilon}}^{\prime}(t)\right) d t+\sigma / 2 \leq \int_{a}^{b} L\left(x(s), x^{\prime}(s)\right) d s-\sigma / 2 .
$$

This contradicts the fact that $x($.$) is a solution to P$. Hence the set $S_{\Lambda}$ has measure zero, and $x($.$) is Lipschitzian on [a, b]$.

Theorem 4 admits the following local version:

Theorem 5. Under the same assumptions on $L$ as in Theorem 4 , let $x($.$) be a$ solution to Problem $(P)$ and let $I \subset[a, b]$ be a compact nontrivial interval such that $(G A)$ is satisfied at every $x(t), t \in I$. Then $x($.$) is Lipschitzian on I.$ 
Example. Consider the problem quoted in the introduction, i.e., the problem of miminizing

$$
\int_{0}^{1}(x(t))^{2}\left(x^{\prime}(t)\right)^{2} d t, \quad x(0)=0, x(1)=1 .
$$

$(G A)$ is not satisfied at $x=0$, and the solution to this problem is $x(t)=\sqrt{t}$. Theorem 5 applied to this case yields that the solution is Lipschitzian on any compact subinterval $I$ of $[0,1]$ such that $t \in I$ implies $x(t) \neq 0$.

\section{REFERENCES}

[A:A:B] L. Ambrosio, O. Ascenzi, and G. Buttazzo, Lipschitz regularity for minimizers of integral functionals with highly discontinuous integrands, J. Math. Anal. Appl. 142 (1989), 301316. MR 91c:49060

[B:M] J. Ball and J. V. Mizel, One-dimensional variational problems whose minimizers do not satisfy the Euler-Lagrange equation, Arch. Rat. Mech. Anal. 90 (1985), 325-388. MR 86k:49002

[C:F:M] A. Cellina, A. Ferriero, and E. M. Marchini, Reparametrizations and approximate values of integrals of the calculus of variations, J. Differential Equations, to appear.

[C:T:Z] A. Cellina, G. Treu, and S. Zagatti, On the minimum problem for a class of non-coercive functionals, J. Differential Equations 127 (1996), 225-262. MR 97d:49003

[Ce] L. Cesari, Optimization, Theory and Applications, Springer-Verlag, New York, 1983. MR 85c:49001

$[\mathrm{C}: \mathrm{V}] \quad$ F. H. Clarke and R. B. Vinter, Regularity properties of solutions to the basic problem in the calculus of variations, Trans. Amer. Math. Soc. 289 (1985), 73-98. MR 86h:49020

[E:T] I. Ekeland and R. Temam, Analyse convexe et problemes variationnels, Dunod, Paris, 1974. MR 57:3931a

[H-U:L] J. B. Hiriart-Urruty and C. Lemarechal, Convex Analysis and Minimization Algorithms. I, Springer-Verlag, Berlin, 1996.

[R] R. T. Rockafellar, Convex Analysis, Princeton Univ. Press, Princeton, 1970. MR 43:445

$[\mathrm{S}: \mathrm{V}] \quad$ J. Serrin and D. E. Varberg, A general chain rule for derivatives and the change of variable formula for the Lebesgue integral, Amer. Math. Monthly 76 (1969), 514-520. MR 40:280

Dipartimento di Matematica e Applicazioni, Università di Milano Bicocca, Via Bicocca Degli Arcimboldi 8, 20126 Milano, Italy

E-mail address: cellina@matapp.unimib.it 